Extraction mit Aether nicht umgangen worden konnte, die directe Extraction der sauren Milch mit Aether ist daher vorzuziehen. Nach dieser Methode ist es nicht schwer, die verschiedenen Pilze auf ihre chemischen Actionen zu prüfen, und die Zersetzungsprodukte zu untersuchen.

Es ist mir gelungen, in der frischen Milch aus Göttingen und Umgegend 5 anscheinend neue Spaltpilze nachzuweisen, welche eine milchsaure Gährung in Mailch und in Nährlösungen mit Zucker bewirken.

Diese 5 Spaltpilze unterscheiden sich mikroskopisch durch ihre Form, makroskopisch durch ihre Reinculturen und physiologisch durch die Art der Milchsäuregährung, dann werden einige durch Aufkochen getodtet, andere nicht. Aus diesen Untersuchungen, über die ich baldigst weiter berichten werde, ergiebt sich, dass es verschiedene Milchsäure-Fermente giebt, welche als geformte Fermente unter den Spaltpilzen angetroffen werden, dass es also nicht ein einheitlicher Pilz ist, welcher uber die Frde verbreitet, so zu sagen, an die Gegenwart der Milch gebunden ist. Die saure Gährung der Milch wird wohl in der Regel durch das Zusammenwirken verschiedener Găhrungsorganismen hervorgerufen, welche vereint eventuell schneller und kräftiger wirken, als eine einzelne Species. Durch dieses Zusammenwirken kommen dann tiefer eingreifende Zersetzungen zustande, welche durch eine einzelne Pilzspecies nicht bewirkt worden.

Die Existenz verschiedener Milchsäure-Organismen wird uns aber die Erklärung der im Anfang dieser Besprechung erwähnten abnormen Erscheinungen, welche bei den Milchsäure-Gährungen beobachtet sind, erleichtern.

\title{
Kleine Beiträge zur Geschichte der Pharmacie.
}

Von A. Conrady, d. Z. in Miltenberg.

I. Unguentum marciaton unter diesem Namen in dem von Fluckiger 1877 herausgegebenen Norrdlinger Register aufgeführt und sich als ungantum marciatori in dem Inventar de 1439 einer Apotheke zu Dijon findend (vergl. Fluckiger Documente, p. 10) scheint bis jetzt räthselhaft geblieben zu sein. Bei der Bearbeitung eines 
sehr interessanten Synonymen-Registers des 15. Jahrhunderts bekam ich nun zufällig 2 Werke in die Hand, die moines Wissens als Quellen bis jetzt nicht genannt wurden. Es sind dies Pharmacia el. Chymia medico physica. Jacobi le mort. Lugd. Batav. 1684, sowic Woytii Gazophyllaceum medicophysicum. Leipzig 1761.

In le morts Werk finde ich unter dem Titel unguentum martiatum nachstehende Vorschrift:

R. Herb. Absinthii; Abrotani; Basilici;

Calaminthae; Contaurii minoris;

Chamädrios; Costi hortensis;

Ilecebrae; Majorane; Mente aquatice

Paralysis; Sabine; Salvie; Sambuci aa $\xi I$

Lauri fol; Rute; Rorismarini aa

Sem. cumini; Fönugräci; Urtice majoris aa $\xi \beta$.

Adipis ursini Medulle cervine

Styrac. liquide $\widehat{a a}$ ZIII

Butyri $\not 6$. I

Ol. Nuciste expressi $\bar{\zeta} \mathrm{I}$

Cere citrine tb I

Vini generosi q. s.

Macerentur peroctidium tepido B.M dein leviter coquantur ad humoris absumptionem ut. f. s. a. ungt.

Vires: Efficax est unguentum in quibuscunque morbis externis e frigore et obstructione oriundis. Vermes necat. Ventriculum roborat.

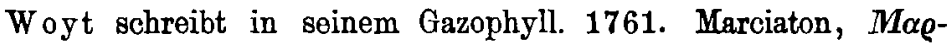
xíatov ist der Nahme einer Salbe vor die Gliederschmerzen, beim Aeginet. 1. 7. c. 18. - Abgesehen von der Diplomatikern so bekannten ähnlichen Schreibweise von $\mathrm{c} u$. $t$ dürften diese Zeilen zur Aufklärung genăgen, da Woyt es aus dem Griechischen ableitet.

II. Pulvis ad vulnera, gleichfalls im Nördlinger Register enthalten und ohne Erklärung geblieben. Woyt giebt hierzu folgende 2 Vorschriften, die wohl auch mit dem P. a. v. des N. Registers nahezu identisch sein dürften:

Pulvis vulnerarius, Wundpulver, das Blut zu stillen.

I. Rp. Farin. vol. 3 II

Bol. armen 
Colofon.

Gypsi $\widehat{\text { å }} \bar{\zeta} \beta$

Alum. ust.

Sang. dracon.

Vitriol. alb. â $3 \pi$

Calcis viv. $3 \mathrm{I}$

Pilor. Lepor. $\boldsymbol{\zeta} \beta$ f. pulv.

II. Rp. Mastich.

Myrrh.

Thuris aa $\bar{\zeta} \beta$

Aloes hepat 3 II

Corall. rubr. praep. ZII f. pulv.

III. Marcasita. Das Vorkommen des Namens Marcasita für verchiedene Metallerze dürfte bereits allgemeiner bekannt geworden sein, wenigstens führt Schmidt in seinem ausführlichen Lehrbuche der ph. Chemie diese Bezeichnung bei Bismuth auf. Flückiger erklärt sowohl in dem Nördlinger Register, als auch in der Esslinger Taxe des 16. Jahrhunderts (Documente) Marcasita; Marchasita auri et argenti, vermuthlich durch die Epitheta verführt, fur Bleiglätte. Dem möchte ich gegenüberstellen, dass im Nördlinger Register ebenfalls litargyrum auri et argenti sich findet und wohl kaum der Verfasser an ein Synonymenverzeichniss dachte, sondern nur eine series medicaminum geben wollte. Die beiden bereits oben angefuhrten Werke le morts und Woyt's dürften unzweifelhaft erweisen, dass es sich gerade hier um Wismuth, resp. um basische Wismuthrerbindungen handelt, (vergl. Schmidt, Chomie I. 320) die damals fast ausschliesslich cosmetischen Zwecken dienten. Das folgen von Bezoleti in der Esslinger Taxe, legt also an und für sich diese Ansicht nahe.

Le mort. Chymia p. 248 schreibt:

Rec. Marcasit. argent. ऊँIII

Aque fortis $\zeta$ VI. f. magisterium.

Marcasitae argenteae.

Dissolvatur marcasita leni cinerum calore, solutionem magnae quantitati depuratae aquae putealis infunde, sic lactescet et sensim ac sensim pulvis deciduus apparebit ad fundum instar Nivis albus et levis, a salium ulteriori consortio diligenter aqua pura cluendus, 
postea inter duas papyros in umbra exsiccandus ad usus cosmeticos. Notae:

1) Aqua putealis ob salis muriatici levioris mixturam praecipitationem facilitat, depuratur autem per coctionem seu subsidentiam, ne faecibus suis albedinem pulveris commaculet.

2) Pulvis hic est levissimus quoniam pedet enim nullo sale pellente descendit, quae levitat et albedo in cosmetico valde commendantur, ut omnes asperitates cutis tollat, loculosque tegat et repleat.

3) Post sabsidentiam prioris pulveris alter in majori copia albus praecipitari potest cum muria salis communis ut in mercurio albo praecipitato proceditur. Vires: Vomitum movet sed rari usus interni est, externe facili et cuti dealbandae inservit cum axungiis vel mucilaginibus, quae multo praestantiores sunt. -

Woyt unterscheidet hauptsächlich Marcasita aurea Goldkiess, Marcasita argentea Silbermarcasit, beide identificirt er und kommt auf Kupfermarcasit oder Pyrites. "Die bekannteste unter allen," schreibt er weiter, ,ist die Zinnmarcasit oder Wismuth" genannt. .... Hiervon werden Magister. und Flores Marcasitae zum Schminken bereitet. Wir sehen also, dass dieso Marcasitae fast alle identisch sind und unzweifelhaft nur für Wismuth, sicher aber nicht für Bleiglätte zu halten sind. -

Später habe ich vielleicht noch Gelegenheit, an der Hand meines neuesten unedirton Synonymenverzeichnisses (Simplicium), das der Zeit des Nördlinger Registers angehören dürfte, mancho Angaben aus Flückigers verdienstvollen Arbeiten zu commentiren oder zu bestätigen.

\section{B. Monatsbericht.}

\section{Pharmaceutische Chemie.}

Nachweis ron Verfulschungen im Extractum Belladonnae. - Ot to Schweissinge $r$ hat die Thatsache, dass Extractum Belladonnae keinen die Fehling'sche Lösung reducirenden Zucker, überhaupt keine oder doch nur höchst geringe Mengen reducirender Substanzen enthält, dazu benutzt, um Verfälschungen des Belladonnaextracts nachzuweisen. Behufs Ausführang der Prüfung wird 1 com Fehling'soher Lösung mit $4 \mathrm{ccm}$ Wasser verdünnt, im Wasserbade auf $60-70^{\circ}$ erhitzt und hierauf etwa $5 \mathrm{cg}$ Extraot (5 Tropfen einer Lösung von $1 \mathrm{~g}$ Extract in $4 \mathrm{~g}$ Wasser) hinzugefügt. Die anfangs blaue Farbe geht in ejne hellgrüne oder hellbraungrüne über und bei echtem Extraot bleibt diese Farbe nach Stunden, ja selbst naoh Tagen unverändert; ist dagegen das Extract verfalsoht, so bildet sich je naoh dem Grade und der Art der Verfalschung eine röthliche Trübung und nach einiger Zeit eine starke Abschei- 